\title{
Portfolio dominance and optimality in infinite security markets
}

\author{
C.D. Aliprantis ${ }^{\text {a,b,c }}$, D.J. Brown ${ }^{\text {d }}$, I.A. Polyrakis ${ }^{\mathrm{e}}$, \\ J. Werner ${ }^{\mathrm{f}, \mathrm{g}, *}$ \\ ${ }^{\mathrm{a}}$ Departments of Mathematics and Economics, IUPUI, USA \\ ${ }^{\mathrm{b}}$ Department of Economics, Cornell University, Ithaca, NY, USA \\ ${ }^{\mathrm{c}}$ Division of Humanities and Social Sciences, CALTECH, Pasadena, CA, USA \\ ${ }^{\mathrm{d}}$ Department of Economics, Yale University, New Haven, CT, USA \\ e Department of Mathematics, National Technical University, Athens, Greece \\ ${ }^{\mathrm{f}}$ Department of Economics, University of Minnesota, 271 19th Avenue South, Minneapolis, MN 55455, \\ USA \\ g Department of Economics, University of Bonn, Bonn, Germany
}

Received 1 October 1996

\begin{abstract}
The most natural way of ordering portfolios is by comparing their payoffs. A portfolio with payoff higher than the payoff of another portfolio is greater in the sense of portfolio dominance than that other portfolio. Portfolio dominance is a lattice order if the supremum and the infimum of any two portfolios are well-defined. We study security markets with infinitely many securities and arbitrary finite portfolio holdings. If portfolio dominance order is a lattice order and has a Yudin basis, then optimal portfolio allocations and equilibria in security markets do exist. (C) 1998 Elsevier Science S.A. All rights reserved.
\end{abstract}

JEL classification: D41; D52; G11; G22

Keywords: Security markets; Portfolio dominance; Equilibrium; Yudin basis

\footnotetext{
* Corresponding Author. Department of Economics, University of Minnesota, 271 19th Avenue South, Minneapolis, MN 55455, USA.
} 


\section{Introduction}

In competitive security markets investors choose portfolios of securities so as to maximize their preferences given prices of the securities. An equilibrium is achieved if investors' optimal portfolios are market clearing. A model of competitive security markets with finitely many securities is due to Hart (1974) (see also Hammond, 1983; Nielsen, 1989; Page, 1987; Werner, 1987 among others) and includes the classical Capital Asset Pricing Model as a special case. Formally, the model is very similar to the standard model of competitive commodity markets. In particular, the space of portfolios plays a role similar to the role of a commodity space. Equilibrium theory of security markets exploits this similarity and relies on the methods of the Arrow-Debreu equilibrium theory. An extensive discussion of the relationship between the Hart's model and the Arrow-Debreu model can be found in Milne (1976) (see also Milne, 1987).

An important difference between a portfolio space and a commodity space is in the order structure of the spaces. While the usual component-wise order is the most relevant for the commodity space, it is of secondary importance for the portfolio space. Far more important is an order induced by the payoff of a portfolio.

Each portfolio is associated with a payoff an investor expects to receive when holding the portfolio. Typically, the payoff is a random consumption stream an element of a payoff space. The mapping that associates a payoff with a portfolio is the payoff operator. The usual order of the payoff space induces via the payoff operator an order on the portfolio space. According to that order one portfolio is greater than another portfolio, if its payoff is higher in every state of the world than the payoff of the other portfolio. We call that order the portfolio dominance order. In general, the portfolio dominance order differs from the component-wise order of the portfolio space. Typically there are portfolios with negative holdings of some securities that have positive payoff in every state.

The cone of positive portfolios (i.e., the positive orthant) under the portfolio dominance order is the set of portfolios with positive payoffs-the most natural set of investor's feasible portfolios. Monotonicity of investors' preferences with respect to the portfolio dominance order is an expression of the desirability of higher consumption.

The portfolio dominance order is a lattice order if for any two portfolios there is a well-defined supremum portfolio and an infimum portfolio. The supremum of two portfolios is the least upper bound with respect to the portfolio dominance order, i.e., a portfolio with the lowest payoff that is higher than the payoffs of both portfolios. The infimum is the greatest lower bound with respect to the portfolio dominance order, i.e., a portfolio with the highest payoff that is lower than the payoffs of both portfolios.

There is an interesting connection between the lattice operations and some important portfolio investment strategies. The supremum of a reference portfolio 
and a portfolio consisting of $k$ shares of a riskless security represents portfolio insurance. It is a portfolio with the least payoff larger than the payoff of the reference portfolio and the floor $k$. If full portfolio insurance is possible so that there is a portfolio with payoff equal to the payoff of the reference portfolio whenever it is above the floor $k$ and equal to $k$ otherwise, then that portfolio equals the supremum of the reference portfolio and $k$ shares of the riskless security relative to the portfolio dominance order.

The lattice order property of the portfolio dominance is therefore of fundamental nature. However, not every set of securities in the Hart's model of security markets generates a portfolio dominance with the lattice order property. The portfolio dominance is a lattice order if and only if the asset span (i.e., the subspace of payoffs of all portfolios) is a lattice subspace of the payoff space. A characterization of security payoffs that span a lattice-subspace can be found in Abramovich et al. (1994) and Polyrakis (1996). A detailed analysis of finite security markets with emphasis on portfolio insurance when security payoffs span a lattice-subspace can be found in Aliprantis et al. (1996b).

In this paper, we consider the case when there are infinitely many securities available for trade. Models of security markets with infinitely many securities are a popular framework for general equilibrium analysis of security pricing; see Brown and Werner (1995). We assume that an investor can hold a portfolio consisting of an arbitrary but finite subset of securities. Since there are only finitely many investors, there is a finite number of securities traded in equilibrium. This finite set of actively traded securities is endogenously determined in equilibrium. Needless to say, all securities are priced in equilibrium. The asset span with such portfolios is the linear span of the securities payoffs in the payoff space.

When an infinite number of securities is available for trade, it is possible that optimal portfolio allocations do not exist. In Section 5 we present an example of security markets where there are infinitely many Arrow securities and a riskless bond available for trade, and there are no optimal portfolio allocations for two risk-averse expected utility maximizing investors.

A crucial property of the portfolio dominance order turns out to be that the positive cone of the portfolio space under the portfolio dominance order has a Yudin basis, or equivalently, that the positive cone of the asset span has a Yudin basis. Yudin basis of the positive cone of the asset span is a set of positive payoffs such that each payoff in the asset span is a linear combination of the payoffs of the basis and that a payoff is positive if and only if it is a positive linear combination of the payoffs of the basis. If an asset span has a Yudin basis, then it is a lattice-subspace, and the portfolio dominance order is a lattice order. The simplest example of a Yudin basis are Arrow securities. More generally, any set of positive payoffs such that for each payoff there is a state in which this payoff is strictly positive while all other payoffs are zero, forms a Yudin basis of the positive cone of its span. More characterizations of Yudin bases can be found in Polyrakis (1996). We emphasize that it is only required that the positive cone of the asset 
span have a Yudin basis and not that the security payoffs themselves form a Yudin basis.

If the asset span has a Yudin basis, optimal portfolio allocations do exist. Furthermore, there exist equilibria in security markets under the standard continuity and convexity assumptions. Our result establishing the existence of optimal portfolio allocations is a significant contribution to the recent literature on equilibrium models of infinite security markets. In Brown and Werner (1995) and Dana et al. (1994) the existence of optimal portfolio allocations (more precisely, the closedness of the utility set) is assumed, not derived from primitive assumptions on agent's preferences or on the securities payoffs. When markets are incomplete, as is the case of their model and the model of this paper, the existence of optimal portfolio allocations has only been verified in special cases. Chichilnisky and Heal (1993), Cheng (1991), Dana and Le Van (1996), and Ishimoto (1996) assumed that the payoff space is a Sobolev space and restricted the class of agents' utility functions. Connor (1984) and Werner (1997) show that optimal portfolio allocations lie in a finite dimensional subspace of the portfolio space when security payoffs have a factor structure as in the Arbitrage Pricing Theory of Ross (1976).

The property of the asset span having a Yudin basis has a simple interpretation. Each payoff of the Yudin basis is a payoff of some portfolio involving finitely many securities. These portfolios can be thought of as mutual funds. Trading mutual funds provides investors with the same spanning opportunities as trading securities. Since investors are only interested in positive payoffs, they have no need to sell short mutual funds. In other words, trading securities is equivalent to trading mutual funds under the no short sales restriction.

The paper is organized as follows: In Section 2 we present basic facts about the mathematical notions of a Yudin basis and a lattice-subspace. In Section 3 we explore the portfolio dominance order of the portfolio space and its connections with portfolio insurance. Optimal portfolio allocations and equilibria in security markets are studied in Sections 4-6. In Section 7 we show a fundamental invariance of our results to a change of security payoffs as long as the asset span remains the same. Using that invariance results we derive the mutual funds interpretation of the Yudin basis. Section 8 presents some interesting results concerning a duality between portfolio allocations and consumption allocations in our model of security markets.

\section{Yudin bases and lattice-subspaces}

As mentioned in the introduction, this work is based heavily on the mathematical notions of a Yudin basis and a lattice-subspace. We shall discuss here briefly the basic properties of these concepts. For details and proofs we refer the reader to 
Aliprantis et al. (1996a). We follow the notation and terminology of the monographs Aliprantis and Border (1994), Aliprantis and Burkinshaw (1978, 1985), and Luxemburg and Zaanen (1971).

A partially ordered vector space $\mathrm{X}$ (whose positive cone is denoted by $\mathrm{X}^{+}$) is said to be a vector lattice or a Riesz space if for every pair of vectors $\mathrm{x}, \mathrm{y} \in \mathrm{X}$ their supremum (least upper bound) and infimum (greatest lower bound) exist in $\mathrm{X}$.

As usual, the supremum and infimum of a pair of vectors $\mathrm{x}, \mathrm{y}$ in a vector lattice are denoted by $\mathrm{x} \vee \mathrm{y}$ and $\mathrm{x} \wedge \mathrm{y}$ respectively. The positive, negative, and absolute value of $x$ are the elements $x^{+}=x \vee 0, x^{-}=(-x) \vee 0$ and $|x|$ respectively. $A$ vector subspace $\mathrm{Y}$ of a vector lattice $\mathrm{X}$ is said to be:

1) a vector sublattice if for each $\mathrm{x}, \mathrm{y} \in \mathrm{Y}$ we have $\mathrm{x} \in \mathrm{Y}$ and $\mathrm{x} \wedge \mathrm{y}$ in $\mathrm{Y}$; and

2) an ideal if $|\mathrm{y}| \leq|\mathrm{x}|$ and $\mathrm{x} \in \mathrm{Y}$ imply $\mathrm{y} \in \mathrm{Y}$. An ideal is always a vector sublattice but a vector sublattice need not be an ideal.

Definition 2.1: A vector subspace $\mathrm{Y}$ of a partially ordered vector space $\mathrm{X}$ is said to be a lattice-subspace if $\mathrm{Y}$ under the induced ordering from $\mathrm{X}$ is a vector lattice in its own right. That is, $\mathrm{Y}$ is a lattice-subspace if for every $\mathrm{x}, \mathrm{y} \in \mathrm{Y}$ the least upper bound of the set $\{\mathrm{x}, \mathrm{y}\}$ exists in $\mathrm{Y}$ when ordered by the cone $\mathrm{Y} \cap \mathrm{X}^{+}$.

If $\mathrm{X}$ is a vector lattice, then every vector sublattice of $\mathrm{X}$ is automatically a lattice-subspace but a lattice-subspace need not be a vector sublattice. For details about lattice-subspaces see Abramovich et al. (1994), Miyajima (1983), and Polyrakis $(1994,1996)$.

A normed space which is also a partially ordered vector space is a partially ordered normed space. A $\|\cdot\|$ norm on a vector lattice is said to be a lattice norm if $|\mathrm{x}| \leq|\mathrm{y}|$ implies $\|\mathrm{x}\| \leq\|\mathrm{y}\|$. A normed vector lattice is a vector lattice equipped with a lattice norm. A complete normed vector lattice is called a Banach lattice.

We are now ready to introduce the notion of a Yudin basis.

Definition 2.2: A cone $C$ of a vector space is called a Yudin cone if there exists a family $\left\{\mathrm{e}_{i}\right\}_{i \in I}$ of vectors of $C$ such that each $\mathrm{x} \in C$ has a unique representation of the form $\mathrm{x}=\sum_{i \in I} \lambda_{i} \mathrm{e}_{i}$, where $\lambda_{i} \geq 0$ and $\lambda_{i}=0$ for all but finitely many $i$. Any such a family $\left\{\mathrm{e}_{i}\right\}_{i \in I}$ of vectors of $C$ is called a Yudin basis of $C$.

Clearly, every Yudin basis of a cone $C$ is a family of linearly independent vectors and is a Hamel basis for its linear span $M=C-C$. In addition, a given cone can have (essentially) at most one Yudin basis (Aliprantis et al., 1996a, Lemma 3.3).

Any subspace of a partially ordered vector space that has a Yudin basis is a lattice-subspace. For finite dimensional subspaces we have the following. 
Lemma 2.1: A finite dimensional vector subspace $M$ of a partially ordered vector space $\mathrm{X}$ is a lattice-subspace if and only if the cone $M^{+}=M \cap \mathrm{X}^{+}$is generating (i.e., $M=M^{+}-M^{+}$) and has a Yudin basis.

However, a positive cone of an infinite dimensional subspace need not have a Yudin basis even if it is a lattice cone. In particular, the positive cone of an infinite dimensional partially ordered vector space with an order unit does not have a Yudin basis (see Aliprantis et al., 1996a, Theorem 3.4). Recall that a positive vector $\mathrm{e}$ is an order unit if for each $\mathrm{x} \in \mathrm{X}$ there exists some $\lambda>0$ satisfying $\mathrm{x} \leq \lambda \mathrm{e}$. Examples of vector spaces with order unit include $\ell_{\infty}, L_{\infty}[0,1], C[0,1]$, and many others.

An important Riesz space for our economic model is the space $\phi$ of all eventually zero real sequences. That is,

$$
\phi=\left\{\boldsymbol{\theta}=\left(\boldsymbol{\theta}_{1}, \boldsymbol{\theta}_{2}, \boldsymbol{\theta}_{3} \ldots\right) \in \mathbb{R}^{\infty}: \boldsymbol{\theta}_{n}=\mathbf{0} \text { for all but a finite number of } n\right\},
$$

where $\mathbb{R}^{\infty}$ denotes the Riesz space of all real sequences. The ordering and the lattice operations in $\phi$ are the pointwise ones. Moreover, $\phi$ equipped with the sup norm, defined by $\|\boldsymbol{\theta}\|_{\infty}=\sup _{n}\left|\boldsymbol{\theta}_{n}\right|$ is a Dedekind complete normed Riesz space. Order intervals in $\phi$ lie in finite dimensional subspaces and are norm compact.

The following fundamental result describes the lattice structure of a vector space generated by a cone with a countable Yudin basis.

Theorem 2.1: Let $C$ by a cone in a vector space $\mathrm{X}$ having a countable Yudin basis $\left\{\mathrm{e}_{n}\right\}$ and let $M=C-C$ be the linear span of $C$. Then we have the following properties.

1. The partially ordered vector space $(M, C)$ is a Dedekind complete Riesz space. If $\mathrm{x}=\sum_{n=1}^{\infty} \lambda_{n} \mathrm{e}_{n}$ and $\mathrm{y}=\sum_{n=1}^{\infty} \mu_{n} \mathrm{e}_{n}$ are arbitrary elements of $M$, then $\mathrm{x} \geq \mathrm{y}$ (i.e., $\mathrm{x}-\mathrm{y} \in C$ ) is equivalent to $\lambda_{n} \geq \mu_{n}$ for each $n$ and the lattice operations of $(M, C)$ are given by

$$
\mathrm{x} \vee \mathrm{y}=\sum_{n=1}^{\infty}\left(\lambda_{n} \vee \mu_{n}\right) \mathrm{e}_{n} \text { and } \mathrm{x} \wedge \mathrm{y}=\sum_{n=1}^{\infty}\left(\lambda_{n} \wedge \mu_{n}\right) \mathrm{e}_{n}
$$

2. The order intervals of $M$ lie in finite dimensional subspaces and hence they are norm compact.

The inductive limit topology on a vector space $M$ is the finest locally convex topology $\xi_{M}$ on $M$ such that for each finite dimensional vector subspace $F$ of $M$ equipped with its Euclidean topology the natural embedding $i: F \hookrightarrow\left(M, \xi_{M}\right)$ is continuous. The inductive limit topology on $\phi$ will be denoted by $\xi$. 
The next remarkable result indicates how one can use a countable family of independent positive vectors to 'twist' the standard lattice ordering of $\phi$. This is a basic result for our work here.

Theorem 2.2: Let $\left\{\mathrm{x}_{n}\right\}$ be a sequence of linearly independent positive vectors in a partially ordered vector space $\mathrm{X}$ and let $M$ denote the span of $\left\{\mathrm{x}_{n}\right\}$. Also, let $R: \phi \rightarrow M$ be the operator defined by $R(\boldsymbol{\theta})=\sum_{n=1}^{\infty} \boldsymbol{\theta}_{n} \mathrm{x}_{n}$. If the cone $M^{+}=M \cap \mathrm{X}^{+}$ has a Yudin basis (which must be necessarily countable), then we have the following.

1. $M$ is a lattice-subspace of $\mathrm{X}$.

2. The vector space $\phi$ equipped with the cone

$$
\phi_{R}^{+}=R^{-1}\left(M^{+}\right)=\{\boldsymbol{\theta} \in \phi: R(\boldsymbol{\theta}) \geq 0\}
$$

is a Dedekind complete Riesz space.

3 . The cone $\phi_{R}^{+}$is a Yudin cone.

4. Each order interval of $\left(\phi, \phi_{R}^{+}\right)$is $\xi$-compact and lies in a finite dimensional vector subspace.

5. The inductive limit topology $\xi$ on the Riesz space $\left(\phi, \phi_{R}^{+}\right)$is Hausdorff, locally convex-solid and order continuous and the operator $R:\left(\phi, \phi_{R}^{+}, \xi\right) \rightarrow\left(M, M^{+}, \xi_{M}\right)$ is a (surjective) topological lattice isomorphism.

6. The Riesz space $\mathbb{R}^{\infty}$ coincides with the topological, algebraic and order dual of $\left(\phi, \phi_{R}^{+}, \xi\right)$. Moreover, $\mathbb{R}^{\infty}$ equipped with the dual cone

$$
\begin{aligned}
\left(\phi_{R}^{+}\right)^{\prime} & =\left\{\mathrm{q}=\left(\mathrm{q}_{1}, \mathrm{q}_{2} \ldots\right) \in \mathbb{R}^{\infty}: \mathrm{q} \cdot \boldsymbol{\theta}=\sum_{n=1}^{\infty} \mathrm{q}_{n} \boldsymbol{\theta}_{n} \geq 0 \forall \boldsymbol{\theta}\right. \\
& \left.=\left(\boldsymbol{\theta}_{1}, \boldsymbol{\theta}_{2}, \ldots\right) \in \phi_{R}^{+}\right\}
\end{aligned}
$$

is a Dedekind complete Riesz space.

7. If $E=\left(\phi, \phi_{R}^{+}\right)$and $E^{\prime}=\left(\mathbb{R}^{\infty},\left(\phi_{R}^{+}\right)^{\prime}\right)$, then $\left\langle E, E^{\prime}\right\rangle$ is a symmetric Riesz dual system.

8. In case $\left\{\mathrm{x}_{n}\right\}$ is itself a Yudin basis, then $\phi_{R}^{+}=\phi^{+}$(the positive cone of $\phi$ under the pointwise order) and $R$ is essentially the identity operator.

\section{Portfolio dominance}

We consider a model of security markets extending over two dates. There are countably many securities traded at date 0 labelled by the natural numbers 1,2 , .... Securities are described by their payoffs at date 1 . The payoff of security $n$ is $\mathrm{x}_{n}$, an element of a payoff space $\mathrm{X}$. The space $\mathrm{X}$ is a partially ordered vector space. Typically, it is a space of real-valued random variables on some underlying probability measure space $(\Omega, \Sigma, P)$, such as a $L_{p}(\Omega, \Sigma, P)$-space for $1 \leq p \leq \infty$. 
Securities can be combined in portfolios. A portfolio is a sequence of share holdings $\boldsymbol{\theta}=\left(\boldsymbol{\theta}_{1}, \boldsymbol{\theta}_{2}, \ldots\right)$, where $\boldsymbol{\theta}_{n}$ is the number of shares of security $n$. In the case of a short position in security $n$, the holding $\boldsymbol{\theta}_{n}$ is negative. Throughout this paper we restrict our attention to portfolios with non-zero holdings of only finitely many securities. Thus, each portfolio is formed from a finite subset of securities. ${ }^{1}$ The space of all portfolios is the vector space $\phi$ of all eventually zero sequences, and will be referred to as the portfolio space.

The payoff of a portfolio $\boldsymbol{\theta} \in \boldsymbol{\phi}$ is

$$
R(\boldsymbol{\theta})=\sum_{n=1}^{\infty} \boldsymbol{\theta}_{n} \mathrm{x}_{n} .
$$

Clearly, $R(\boldsymbol{\theta}) \in \mathrm{X}$. It should be clear that this formula defines a linear operator $R$ : $\phi \rightarrow \mathrm{X}$, which we shall refer to as the payoff operator. The payoff vectors $\mathrm{x}_{1}, \mathrm{x}_{2}$, etc. are assumed to be linearly independent (non-redundant securities), so that the payoff operator $R$ is always one-to-one.

The partial order of the payoff space $\mathrm{X}$ induces a partial order $\geq_{R}$ on the portfolio space $\phi$ via the payoff operator $R$ by

$$
\boldsymbol{\theta} \geq{ }_{R} \boldsymbol{\theta}^{\prime} \text { whenever } R(\boldsymbol{\theta}) \geq R\left(\boldsymbol{\theta}^{\prime}\right) .
$$

The order $\geq_{R}$ will be called the portfolio dominance order. ${ }^{2}$ The positive cone under the portfolio dominance order is

$$
\phi_{R}^{+}=\left\{\boldsymbol{\theta} \in \phi: \boldsymbol{\theta} \geq_{R} 0\right\}=\{\boldsymbol{\theta} \in \phi: R(\boldsymbol{\theta}) \geq 0\}=R^{-1}\left(\mathrm{X}^{+}\right)
$$

and is precisely the set of all portfolios with positive payoffs. The cone $\phi_{R}^{+}$will be referred to as the cone of positive payoff portfolios. As usual, $\boldsymbol{\theta} \geq{ }_{R} \boldsymbol{\theta}^{\prime}$ if and only if $\boldsymbol{\theta}-\boldsymbol{\theta}^{\prime} \in \phi_{R}^{+}$.

We shall assume that the portfolio dominance order $\geq_{R}$ is a lattice order. That is, for any two portfolios $\boldsymbol{\theta}, \boldsymbol{\theta}^{\prime} \in \boldsymbol{\phi}$ we have a well-defined supremum portfolio $\boldsymbol{\theta} \vee{ }_{R} \boldsymbol{\theta}^{\prime}$, and an infimum portfolio $\boldsymbol{\theta} \wedge{ }_{R} \boldsymbol{\theta}^{\prime}$. The supremum $\boldsymbol{\theta} \vee{ }_{R} \boldsymbol{\theta}^{\prime}$ is the least upper bound of $\boldsymbol{\theta}$ and $\boldsymbol{\theta}^{\prime}$ with respect to $\geq_{R}$, i.e., a portfolio with the lowest payoff that is higher than the payoffs of $\boldsymbol{\theta}$ and $\boldsymbol{\theta}^{\prime}$. The infimum $\boldsymbol{\theta} \vee{ }_{R} \boldsymbol{\theta}^{\prime}$ is the greatest lower bound of $\boldsymbol{\theta}$ and $\boldsymbol{\theta}^{\prime}$ with respect to $\geq_{R}$, i.e., a portfolio with the highest payoff that is lower than the payoffs of $\boldsymbol{\theta}$ and $\boldsymbol{\theta}^{\prime}$.

There is an interesting connection between lattice operations and some important portfolio investment strategies. Suppose that the payoff space is $\mathrm{X}=$ $L_{p}(\Omega, \Sigma, P)$, and that security 1 is a riskless security with payoff $\mathrm{x}_{1}(\omega)=1$ for

\footnotetext{
${ }^{1}$ Such portfolios might be called simple portfolios in analogy with the simple trading strategies in the continuous time model of security markets of Harrison and Kreps (1979).

${ }^{2}$ We use the subscript $R$ to distinguish this linear ordering from the standard pointwise ordering $\geq$ on $\phi$ defined by $\boldsymbol{\theta} \geq \boldsymbol{\theta}^{\prime}$ whenever $\boldsymbol{\theta}_{n} \geq \boldsymbol{\theta}_{n}^{\prime}$ for each $n$.
} 
every $\omega \in \Omega$. Let $\mathrm{e}_{1}=(1,0,0, \ldots) \in \phi$ denote the portfolio of one share of the riskless security. For any reference portfolio $\boldsymbol{\theta}$ and any positive number ('floor') $k$, the portfolio $\boldsymbol{\theta} \vee{ }_{R} k \mathrm{e}_{1}$ is the portfolio with the lowest payoff higher than the payoff of $\boldsymbol{\theta}$ and the floor $k$. Thus the portfolio $\boldsymbol{\theta} \vee{ }_{R} k \mathrm{e}_{1}$ represents portfolio insurance. If there exists a portfolio the payoff of which equals the payoff of $\boldsymbol{\theta}$ whenever the latter is above the floor $k$ and equals $k$, otherwise, then that portfolio equals $\boldsymbol{\theta} \vee{ }_{R} \mathrm{e}_{1}$, and we have full portfolio insurance. In a companion paper, Aliprantis et al. (1996b), we provide a detailed analysis of portfolio insurance when the portfolio dominance order is a lattice order.

The range $M=R(\phi) \subseteq \mathrm{X}$ of the payoff operator $R$ is the subspace of payoffs of all portfolios. We shall refer to $M$ as the asset span of the securities-it is also known as the space of marketed securities. By a theorem of Vulikh (Theorem 7.14 in Aliprantis and Border, 1994), for $\geq_{R}$ to be a lattice order it is necessary and sufficient that $M$ is a Riesz space under the induced ordering from $\mathrm{X}$.

Lemma 3.1: The portfolio dominance order $\geq_{R}$ on $\phi$ is a lattice order (or, equivalently, $\phi_{R}^{+}$is a lattice cone of $\left.\phi\right)$ if and only if the asset span $M$ is a lattice-subspace of the payoff space X.

So, assuming that $\phi_{R}^{+}$is a lattice cone is equivalent to asserting that $M^{+}=M$ $\cap \mathrm{X}^{+}$is a lattice cone of the asset span $M$. Conditions under which a subspace is a lattice-subspace will be discussed in Section 5 (see also Abramovich et al., 1994; Polyrakis, 1996).

A price of security $n$ is denoted by $\mathrm{q}_{n}$. Any vector $\mathrm{q}=\left(\mathrm{q}_{1}, \mathrm{q}_{2}, \ldots\right) \in \mathbb{R}^{\infty}$ will be called a security price system or simply a vector of security prices. The market value of portfolio $\boldsymbol{\theta} \in \phi$ at security prices $q$ is then the real number

$$
\mathrm{q} \cdot \boldsymbol{\theta}=\sum_{n=1}^{\infty} \mathrm{q}_{n} \boldsymbol{\theta}_{n} .
$$

The portfolio space $\phi$ and the space of security prices $\mathbb{R}^{\infty}$ form a dual system $\left\langle\phi, \mathbb{R}^{\infty}\right\rangle$, the portfolio-price duality. By Theorem 2.2 , we know that $\left\langle\phi, \mathbb{R}^{\infty}\right\rangle$ is a symmetric Riesz dual system. The dual cone $\left(\phi_{R}^{+}\right)^{\prime}$ of $\phi_{R}^{+}$is defined by

$$
\begin{aligned}
\left(\phi_{R}^{+}\right)^{\prime} & =\left\{\mathrm{q}=\left(\mathrm{q}_{1}, \mathrm{q}_{2}, \ldots\right) \in \mathbb{R}^{\infty}: \mathrm{q} \cdot \boldsymbol{\theta}=\sum_{n=1}^{\infty} \mathrm{q}_{n} \boldsymbol{\theta}_{n} \geq 0 \forall \boldsymbol{\theta}\right. \\
& \left.=\left(\boldsymbol{\theta}_{1}, \boldsymbol{\theta}_{2}, \ldots\right) \in \phi_{R}^{+}\right\} .
\end{aligned}
$$

The standard concepts of arbitrage and strong arbitrage portfolios can be easily expressed using the portfolio dominance. A strong arbitrage under prices $\mathrm{q}$ is a portfolio $\boldsymbol{\theta} \in \boldsymbol{\phi}$ that dominates the zero portfolio $\left(\boldsymbol{\theta} \geq_{R} 0\right)$ and has negative value $(q \cdot \boldsymbol{\theta}<0)$, i.e., a portfolio with negative value and positive payoff. An 
arbitrage under prices $\mathrm{q}$ is a portfolio $\boldsymbol{\theta} \in \boldsymbol{\phi}$ such that $\boldsymbol{\theta}>{ }_{R} 0$ and $\mathrm{q} \cdot \boldsymbol{\theta} \leq \boldsymbol{\theta}$, i.e., a portfolio with zero or negative value and positive and nonzero payoff. A security price system that excludes strong arbitrage (resp. arbitrage) is weakly arbitrage-free (resp. arbitrage-free). Clearly, every strong arbitrage is an arbitrage, and therefore every arbitrage-free price is also weakly arbitrage-free. The set of weakly arbitrage-free prices is the dual cone $\left(\phi_{R}^{+}\right)$.

Since $\left\langle\left(\phi, \phi_{R}^{+}\right),\left(\mathbb{R}^{\infty},\left(\phi_{R}^{+}\right)^{\prime}\right)\right\rangle$ is a Riesz dual system, it follows that

$$
\boldsymbol{\theta} \geq_{R} \boldsymbol{\theta}^{\prime} \Leftrightarrow \mathrm{q} \cdot \boldsymbol{\theta} \geq \mathrm{q} \cdot \boldsymbol{\theta}^{\prime} \text { for each weak arbitrage }- \text { free price vector q. }
$$

Thus, a portfolio $\boldsymbol{\theta}$ dominates another portfolio $\boldsymbol{\theta}^{\prime}$ if and only if $\boldsymbol{\theta}$ is more expensive than $\boldsymbol{\theta}^{\prime}$ under every weakly arbitrage-free price.

\section{Equilibrium in security markets}

In the portfolio-price duality $\left\langle\phi, \mathbb{R}^{\infty}\right\rangle$, the portfolio space $\phi$ is understood as a Riesz space with the lattice cone $\phi_{R}^{+}$, and the price space $\mathbb{R}^{\infty}$ as a Riesz space with the lattice cone $\left(\phi_{R}^{+}\right)^{\prime}$. Unless otherwise stated, $\phi$ will be understood equipped with its inductive limit topology $\xi$.

There are $m$ investors indexed by $i$, i.e., $i=1, \ldots, m$. Each investor $i$ has:

1. The cone of positive payoff portfolios $\phi_{R}^{+}$as her feasible portfolio set.

2. An initial portfolio $\overline{\boldsymbol{\theta}}^{i} \in \phi_{R}^{+}$. The aggregate portfolio $\overline{\boldsymbol{\theta}}=\sum_{i=1}^{m} \overline{\boldsymbol{\theta}}^{i}$ is called the market portfolio.

3. A utility function $\hat{u}_{i}: \phi_{R}^{+} \rightarrow \mathbb{R}$ such that

3.1. $\hat{u}_{i}$ is quasi-concave and $\xi$-continuous, and

3.2. $\hat{u}_{i}$ is monotone with respect to $\geq_{R}$, i.e., $\boldsymbol{\theta} \geq{ }_{R} \boldsymbol{\theta}^{\prime}$ implies $\hat{u}_{i}(\boldsymbol{\theta}) \geq \hat{u}_{i}\left(\boldsymbol{\theta}^{\prime}\right)$.

4. The market portfolio $\overline{\boldsymbol{\theta}}$ is desirable in the sense that $\hat{u}_{i}(\boldsymbol{\theta}+\alpha \overline{\boldsymbol{\theta}})>\hat{u}_{i}(\boldsymbol{\theta})$ for all $\boldsymbol{\theta} \in \phi_{R}^{+}$and each $\alpha>0$.

If an investor $i$ has a preference over the state-contingent consumption plans described by a utility function $u_{i}: \mathrm{X}^{+} \rightarrow \mathbb{R}$, then we shall assume that the portfolio utility function $\hat{u}_{i}$ is the indirect utility given by $\hat{u}_{i}(\boldsymbol{\theta})=u_{i}(R(\boldsymbol{\theta}))$. When $\mathrm{X}=$ $L_{p}(\Omega, \Sigma, P)$, a typical example of a utility function $u_{i}: \mathrm{X}^{+} \rightarrow \mathbb{R}$ is a separable utility function given by

$$
u_{i}(\mathrm{x})=\int_{\Omega} v_{i}(\mathrm{x}(\omega)) \mathrm{d} P(\omega),
$$

where the kernel $v_{i}: \mathbb{R}_{+} \times \Omega \rightarrow \mathbb{R}$ satisfies certain concavity and measurability properties; see Aliprantis (1996b) for details about separable utility functions.

If a consumption utility function $u_{i}: \mathrm{X}^{+} \rightarrow \mathbb{R}$ is quasi-concave, monotone, and the payoff $R(\overline{\boldsymbol{\theta}})$ is desirable for $u_{i}$, then the indirect utility function $\hat{u}_{i}$ is quasi-concave, monotone, and $\overline{\boldsymbol{\theta}}$ is desirable for $\hat{u}_{i}$. Moreover, if $u_{i}$ is continuous 
for a Hausdorff locally convex topology of the payoff space $X$, then $\hat{u}_{i}$ is continuous in the inductive limit topology $\xi$ of the portfolio space $\phi$.

An optimal portfolio for investor $i$ at prices $\mathrm{q}$ is a portfolio $\boldsymbol{\theta}^{i}$ that maximizes the utility $\hat{u}_{i}$ over all portfolios in the budget set

$$
\mathscr{B}_{i}(\mathrm{q})=\left\{\boldsymbol{\theta} \in \phi_{R}^{+}: \mathrm{q} \cdot \boldsymbol{\theta} \leq \mathrm{q} \cdot \overline{\boldsymbol{\theta}}^{i}\right\}
$$

A portfolio allocation is any $m$-tuple $\left(\boldsymbol{\theta}^{1}, \ldots, \boldsymbol{\theta}^{m}\right)$, where $\boldsymbol{\theta}^{i}$ is a feasible portfolio (i.e., $\boldsymbol{\theta}^{i} \in \boldsymbol{\phi}_{R}^{+}$) for investor $i$ and $\sum_{i=1}^{m} \boldsymbol{\theta}^{i}=\sum_{i=1}^{m} \overline{\boldsymbol{\theta}}^{i}=\overline{\boldsymbol{\theta}}$.

An equilibrium in security markets is now defined as follows.

Definition 4.1: A portfolio allocation $\left(\boldsymbol{\theta}^{1}, \ldots, \boldsymbol{\theta}^{m}\right)$ is said to be a portfolio equilibrium if there exists a non-zero security price system $q \in \mathbb{R}^{\infty}$ such that each $\boldsymbol{\theta}^{i}$ is optimal for investor $i$ at prices $\mathrm{q}$.

For studying sufficient conditions for the existence of a portfolio equilibrium it is useful to introduce the notion of a portfolio quasiequilibrium.

Definition 4.2: $A$ portfolio quasiequilibrium is a portfolio allocation $\left(\boldsymbol{\theta}^{1}, \ldots, \boldsymbol{\theta}^{m}\right)$ for which there exists a non-zero price system $q$ such that

$$
\boldsymbol{\theta} \in \phi_{R}^{+} \text {and } \hat{u}_{i}(\boldsymbol{\theta}) \geq \hat{u}_{i}\left(\boldsymbol{\theta}^{i}\right) \text { imply } \mathrm{q} \cdot \boldsymbol{\theta} \geq \mathrm{q} \cdot \overline{\boldsymbol{\theta}}^{i} \text {. }
$$

Clearly, every equilibrium is a quasiequilibrium. Conversely, a quasiequilibrium in which the wealth of each agent is strictly positive is an equilibrium. Every price system that supports a quasiequilibrium is weakly arbitrage-free.

\section{Optimal portfolio allocations}

An optimal portfolio allocation is defined as follows.

Definition 5.1: A portfolio allocation $\left(\boldsymbol{\theta}^{1}, \ldots, \boldsymbol{\theta}^{m}\right)$ is optimal, if there is no other portfolio allocation $\left(\boldsymbol{\theta}^{\prime 1}, \ldots, \boldsymbol{\theta}^{\prime m}\right)$ satisfying $\hat{u}_{i}\left(\boldsymbol{\theta}^{\prime i}\right) \geq \hat{u}_{i}\left(\boldsymbol{\theta}^{i}\right)$ for every $i$ and $\hat{u}_{i}\left(\boldsymbol{\theta}^{\prime i}\right)>\hat{u}_{i}\left(\boldsymbol{\theta}^{i}\right)$ for at least one $i$.

By the First Welfare Theorem a portfolio equilibrium allocation is (under the standard assumptions) optimal. Clearly, it is also individually rational in the sense that it is weakly preferred to the initial portfolio allocation $\left(\overline{\boldsymbol{\theta}}^{1}, \ldots, \overline{\boldsymbol{\theta}}^{m}\right)$. The existence of individually rational optimal portfolio allocations is a necessary condition for the existence of a portfolio equilibrium. 
The utility possibility set is the set of utility levels of individually rational portfolio allocations

$$
\begin{aligned}
U & =\left\{\left(\lambda_{1}, \ldots, \lambda_{m}\right) \in \mathbb{R}^{m}: \exists \text { some allocation }\left(\boldsymbol{\theta}^{1}, \ldots, \boldsymbol{\theta}^{m}\right) \text { with } \hat{u}_{i}\left(\overline{\boldsymbol{\theta}}^{i}\right)\right. \\
& \left.\leq \lambda_{i} \leq \hat{u}_{i}\left(\boldsymbol{\theta}^{i}\right) \forall i\right\} .
\end{aligned}
$$

Monotonicity of the portfolio utility functions implies that the utility set is a bounded set. If it is closed, then the existence of optimal portfolio allocations is assured. Indeed, portfolio allocations that generate the highest (in the sense of the usual order of $\mathbb{R}^{m}$ ) utility levels are optimal, and so their utility allocations lie in the boundary of the utility possibility set.

If $\left(\boldsymbol{\theta}^{1}, \ldots, \boldsymbol{\theta}^{m}\right)$ is a feasible portfolio allocation, then $0 \leq{ }_{R} \boldsymbol{\theta}^{i} \leq{ }_{R} \overline{\boldsymbol{\theta}}$, i.e., each portfolio $\boldsymbol{\theta}^{i}$ lies in the order interval $[0, \overline{\boldsymbol{\theta}}]_{R}$ of the portfolio dominance order. A sufficient condition for the closedness of the utility set is the weak compactness of the order interval $[0, \overline{\boldsymbol{\theta}}]_{R}$; see Aliprantis (1996a) (Problem 3.5.1, p. 145). If the cone of positive payoff portfolios $\phi_{R}^{+}$has a Yudin basis, then it follows from Theorem 2.2, (4) that the interval $[0, \overline{\boldsymbol{\theta}}]_{R}$ lies in the finite dimensional vector subspace-and hence is compact.

We have the following.

Theorem 5.1: If the cone of positive payoff portfolios $\phi_{R}^{+}$has a Yudin basis (or, equivalently, if the positive cone $M^{+}=M \cap \mathrm{X}^{+}$of the asset span $M$ has a Yudin basis), then the utility possibility set $U$ is closed.

Proof: We consider $\phi$ as a Riesz space under the order $\geq_{R}$. As mentioned above, by Theorem $2.2(4)$, the interval $[0, \overline{\boldsymbol{\theta}}]_{R}$ is compact and lies in a finite dimensional subspace of $\phi$.

Let a sequence $\left\{\boldsymbol{\lambda}_{n}\right\} \subseteq U$ satisfy $\boldsymbol{\lambda}_{n} \rightarrow \boldsymbol{\lambda}$ in $\mathbb{R}^{m}$, where $\boldsymbol{\lambda}_{n}=\left(\lambda_{1}^{n}, \ldots, \lambda_{m}^{n}\right)$. For each $n$ pick a portfolio allocation $\left(\boldsymbol{\theta}^{1 n}, \ldots, \boldsymbol{\theta}^{m n}\right)$ such that $\hat{u}_{i}\left(\overline{\boldsymbol{\theta}}^{i}\right) \leq \lambda_{i}^{n} \leq \hat{u}_{i}\left(\boldsymbol{\theta}^{i n}\right)$ for each $i$ and $n$. Since $0 \leq{ }_{R} \boldsymbol{\theta}^{i n} \leq{ }_{R} \overline{\boldsymbol{\theta}}$ for each $i$ and $n$ and the order interval $[0, \overline{\boldsymbol{\theta}}]_{R}$ is compact, by passing to appropriate subsequences if necessary, we can assume that $\boldsymbol{\theta}^{i n} \rightarrow \boldsymbol{\theta}^{i}$ for each $i$. Clearly, $\left(\boldsymbol{\theta}^{1}, \ldots, \boldsymbol{\theta}^{m}\right)$ is a portfolio allocation. The $\xi$-continuity of the utility functions implies

$$
\hat{u}_{i}\left(\overline{\boldsymbol{\theta}}^{i}\right) \leq \lambda_{i}=\lim _{n \rightarrow \infty} \lambda_{i}^{n} \leq \lim _{n \rightarrow \infty} \hat{u}_{i}\left(\boldsymbol{\theta}^{i n}\right)=\hat{u}_{i}\left(\boldsymbol{\theta}^{i}\right) .
$$

This shows $\boldsymbol{\lambda}=\left(\lambda_{1}, \ldots, \lambda_{m}\right) \in U$ so that $U$ is a closed subset of $\mathbb{R}_{+}^{m} \cdot{ }^{3}$

The simplest example of the asset span with a Yudin basis is the span of Arrow securities. More generally, any set of positive payoffs such that for each payoff there is a state in which this payoff is strictly positive while all other payoffs are

\footnotetext{
${ }^{3}$ The reader should notice that this proof also shows that the utility set remains closed if we assume that the utility functions are only $\xi$-upper semicontinuous rather than $\xi$-continuous.
} 
zero, forms a Yudin basis of the positive cone of its span, see Polyrakis (1996). We emphasize that Theorem 5.1 requires only that the positive cone of the asset span have a Yudin basis and not that the security payoffs themselves form a Yudin basis.

The next two examples illustrate the closedness of the utility possibility set.

Example 5.1: Let the securities be the Arrow securities, i.e., $\mathrm{x}_{n}=\mathrm{e}_{n}$ is the $n$th unit vector in the payoff space $\mathrm{X}=\ell_{\infty}$ of all bounded consumption plans on a countable state space. In this case the payoff operator $R: \phi \rightarrow \ell_{\infty}$ is simply the natural embedding of $\phi$ into $\ell_{\infty}$ (i.e., $R(\boldsymbol{\theta})=\boldsymbol{\theta}$ ) and the portfolio dominance order coincides with the usual order of the portfolio space $\phi$, i.e.,

$$
\boldsymbol{\theta} \geq_{R} \boldsymbol{\theta} \Leftrightarrow \boldsymbol{\theta}_{n} \geq \boldsymbol{\theta}_{n}^{\prime} \text { for each } n .
$$

The order interval $[0, \overline{\boldsymbol{\theta}}]$ lies in a finite dimensional vector subspace of $\phi$. Indeed, the set $\mathscr{F}=\left\{n: \overline{\boldsymbol{\theta}}_{n}>0\right\}$ is finite, and $\boldsymbol{\theta} \in[0, \overline{\boldsymbol{\theta}}]$ implies that $\boldsymbol{\theta}_{n}=0$ for $n \notin \mathscr{F}$. Consequently, $[0, \overline{\boldsymbol{\theta}}]$ is compact and the utility possibility set $U$ is closed. In fact, $U$ is the same as the utility possibility set of an economy with the finite set of securities $\left\{\mathrm{x}_{n}: n \in \mathscr{F}\right\}$. Note that the positive cone $\phi_{R}^{+}$has a Yudin basis consisting of the unit vectors.

More generally, if the payoffs $\mathrm{x}_{n}$ are such that for each security $n$ there is a state $\omega_{n} \in \Omega$ in which $\mathrm{x}_{n}\left(\omega_{n}\right)>0$ and $\mathrm{x}_{k}\left(\omega_{n}\right)=0$ for every security $k \neq n$, then the portfolio dominance order coincides with the usual order of $\phi$. The utility possibility set is closed in such a case.

Example 5.2: Let the securities $\left\{\mathrm{x}_{1}, \mathrm{x}_{2}, \ldots\right\}$ be the Arrow securities and $\mathrm{x}_{0}$ be the riskless bond $\mathrm{x}_{0}=\mathrm{e}=(1,1, \ldots)$, where $\mathrm{e}$ is the order unit in the payoff space $\mathrm{X}=\ell_{\infty}$. The payoff operator $R: \phi \rightarrow \ell_{\infty}$ is given by

$$
R(\boldsymbol{\theta})=\left(\boldsymbol{\theta}_{0}+\boldsymbol{\theta}_{1}, \boldsymbol{\theta}_{0}+\boldsymbol{\theta}_{2}, \boldsymbol{\theta}_{0}+\boldsymbol{\theta}_{3}, \ldots\right)
$$

for each $\boldsymbol{\theta}=\left(\boldsymbol{\theta}_{0}, \boldsymbol{\theta}_{1}, \boldsymbol{\theta}_{2}, \ldots\right) \in \phi$. The portfolio dominance order in this case is

$$
\boldsymbol{\theta} \geq{ }_{R} \boldsymbol{\theta}^{\prime} \Leftrightarrow \boldsymbol{\theta}_{0}+\boldsymbol{\theta}_{n} \geq \boldsymbol{\theta}_{0}^{\prime}+\boldsymbol{\theta}_{n}^{\prime} \text { for each n. }
$$

Suppose that the Arrow securities are in zero supply and that there is a strictly positive supply $b$ of the bond. Then the market portfolio $\overline{\boldsymbol{\theta}}$ includes only the bond so that $\overline{\boldsymbol{\theta}}=(b, 0,0, \ldots)$. The order interval $[0, \overline{\boldsymbol{\theta}}]_{R}$ is not $\boldsymbol{\xi}$-compact. Indeed, if we let $\gamma_{n}=b \mathrm{e}_{n}$ for each $n$, then $\gamma_{n}$ lies in the order interval $[0, \overline{\boldsymbol{\theta}}]_{R}$ for each $n$, but the sequence $\left\{\gamma_{n}\right\}$ does not have any $\xi$-convergent subnet.

The asset span $M$ is the subspace of all eventually constant sequences. That subspace is a vector sublattice of $\ell_{\infty}$. In particular it is a lattice-subspace. However, since $M$ is infinite dimensional and has an order unit, the positive cone $M^{+}$does not have a Yudin basis (see Aliprantis et al., 1996a, Theorem 3.4), and so the cone of positive payoff portfolios $\phi_{R}^{+}$does not have a Yudin basis either. 
We shall specify now investors' utility functions for which the utility set is not closed. There are two risk averse expected utility maximizing investors, $i=1,2$, with the consumption utility functions $u_{i}: \ell_{\infty}^{+} \rightarrow \mathbb{R}$ given by

$$
u_{i}(\mathrm{x})=\sum_{\omega=1}^{\infty} P_{i}(\omega) v_{i}(\mathrm{x}(\omega))
$$

where $v_{i}:[0, \infty) \rightarrow \mathbb{R}$ is strictly increasing, strictly concave and continuous. The probability beliefs $P_{i}$ on the state space $\Omega=\{1,2, \ldots\}$ are given by $P_{1}(\omega)=2^{-\omega}$ for every $\omega \in \Omega$, and $P_{2}(\omega)=2^{1-\omega}$, if $\omega \in A=\{2,4,6, \ldots\}$ and $P_{2}(\omega)=2^{-1-\omega}$, if $\omega \in A^{\mathrm{c}}=\{1,3,5, \ldots\}$. Note that investors assign the same conditional probabilities to every state, conditional on $A$ and on $A^{c}$, but they assign different probabilities to events $A$ and $A^{c}$.

The payoff $R(\overline{\boldsymbol{\theta}})=b \mathrm{x}_{0}=(b, b, \ldots)$ of the market portfolio is state independent. We claim that all Pareto optimal consumption allocations with respect to $\mathrm{X}=\ell_{\infty}$ are state independent within $A$ and within $A^{c}$.

In order to prove this claim, consider a Pareto optimal consumption allocation $\left(\mathrm{y}_{1}, \mathrm{y}_{2}\right)$ and suppose that $\mathrm{y}_{1}\left(\omega_{1}\right) \neq \mathrm{y}_{1}\left(\omega_{2}\right)$ for some $\omega_{1}, \omega_{2} \in A$. Define the consumption plans $\overline{\mathrm{y}}_{1}$ and $\overline{\mathrm{y}}_{2}$ by

$$
\bar{y}_{i}(\omega)=\left\{\begin{array}{cc}
\mathrm{y}_{i}(\omega), & \text { if } \omega \notin\left\{\omega_{1}, \omega_{2}\right\} \\
z_{i}, & \text { if } \omega \in\left\{\omega_{1}, \omega_{2}\right\},
\end{array}\right.
$$

where $z_{i}=P_{i}\left(\omega_{1} \mid\left\{\omega_{1}, \omega_{2}\right\}\right) \mathrm{y}_{i}\left(\omega_{1}\right)+P_{i}\left(\omega_{2} \mid\left\{\omega_{1}, \omega_{2}\right\}\right) \mathrm{y}_{i}\left(\omega_{2}\right)$ is the expected value of $\mathrm{y}_{i}$ conditional on $\left\{\omega_{1}, \omega_{2}\right\}$. Since the conditional probabilities $P_{i}\left(\omega_{1} \mid\left\{\omega_{1}, \omega_{2}\right\}\right)$ and $P_{i}\left(\omega_{2} \mid\left\{\omega_{1}, \omega_{2}\right\}\right)$ are the same for both investors, $\left(\bar{y}_{1}, \overline{\mathrm{y}}_{2}\right)$ is an allocation. Moreover, the strict concavity of $v_{i}$ implies

$$
\begin{aligned}
u_{i}\left(\overline{\mathrm{y}}_{i}\right)-u_{i}\left(\mathrm{y}_{i}\right)= & P_{i}\left(\left\{\omega_{1}, \omega_{2}\right\}\right) v_{i}\left(z_{i}\right)-\left[P_{i}\left(\omega_{1}\right) v_{i}\left(\mathrm{y}_{i}\left(\omega_{1}\right)\right)\right. \\
& \left.+P_{i}\left(\omega_{2}\right) v_{i}\left(\mathrm{y}_{i}\left(\omega_{2}\right)\right)\right]>0
\end{aligned}
$$

for each $i=1,2$, which contradicts the Pareto optimality of the allocation $\left(\mathrm{y}_{1}, \mathrm{y}_{2}\right)$.

Next we claim that all Pareto optimal allocations (with the exception of 'corner' allocations where one investor has zero consumption) are state dependent across $A$ and $A^{c}$. This is obvious since the marginal rate of substitution between consumption in any two states $\omega \in A$ and $\omega^{\prime} \in A^{c}$ at a state independent consumption plan equals $\left(P_{i}(\omega)\right) /\left(P_{i}\left(\omega^{\prime}\right)\right)$, and is different for $i=1$ and for $i=2$. Equality of marginal rates of substitution is a necessary condition for Pareto optimality.

Since the asset span $M$ is the space of all eventually constant sequences, Pareto optimal consumption plans do not belong to the asset span $M$. However, they can be approximated by consumption plans in $M$ so that the difference in utility is arbitrarily small. More precisely, let $\left(\mathrm{y}_{1}, \mathrm{y}_{2}\right)$ be a Pareto optimal allocation. Consider the sequence of allocations $\left\{\left(\mathrm{y}_{1}^{n}, \mathrm{y}_{2}^{n}\right)\right\}$ such that $\mathrm{y}_{i}^{n}(\omega)=\mathrm{y}_{i}(\omega)$ for $\omega \leq n$, 
and $\mathrm{y}_{i}^{n}(\omega)=R(\overline{\boldsymbol{\theta}}(\omega)) / 2$ for $\omega>n, i=1,2$. We have that $\mathrm{y}_{i}^{n} \in M$ and $\left\{\mathrm{y}_{i}{ }^{n}\right\}$ converges in the weak topology $\sigma\left(\ell_{\infty}, \ell_{1}\right)$ to ${ }^{i}$. Since the expected utility function $u_{i}$ is weakly continuous on the order interval $[0, R(\overline{\boldsymbol{\theta}})]$ of $\ell_{\infty}$, it follows that $u_{i}\left(\mathrm{y}_{i}^{n}\right)$ converges to $u_{i}\left(\mathrm{y}_{i}\right)$. So, the utility set $U$ is not closed.

\section{Existence of a portfolio equilibrium}

Our analysis of the existence of a portfolio equilibrium follows the approach of Mas-Colell (1986); see also Aliprantis et al. (1990), (Theorem 3.5.12, p. 161).

Suppose that the cone of positive payoff portfolios has a Yudin basis which is, of course, equivalent to saying that the cone $M^{+}=M \cap \mathrm{X}^{+}$of the asset span $M$ has a Yudin basis. Then, by Theorem 5.1, the utility possibility set of security markets is closed. Thus, there exist optimal portfolio allocations, a necessary condition for the existence of an equilibrium. The remaining issue is the existence of the supporting prices, i.e., security prices that would make an optimal portfolio allocation a portfolio quasiequilibrium for a suitable allocation of initial portfolios. That issue-which is specific to infinite dimensional equilibrium theory-is handled by restricting the class of investors' utility functions to those that are also uniformly proper on $\phi_{R}^{+}$.

Recall that a portfolio utility function $\hat{u}_{i}$ is $\overline{\boldsymbol{\theta}}$-uniformly $\xi$-proper on $\phi_{R}^{+}$, if there is a neighborhood (in the inductive limit topology) $V$ of zero such that $\hat{u}_{i}(\boldsymbol{\theta}-\alpha \overline{\boldsymbol{\theta}}+\gamma) \geq \hat{u}_{i}(\boldsymbol{\theta})$ implies $\gamma \notin \alpha V$ for every $\alpha>0$ and $\boldsymbol{\theta} \in \boldsymbol{\phi}_{R}^{+}$with $\boldsymbol{\theta}-\alpha \overline{\boldsymbol{\theta}}+\gamma \in \phi_{R}^{+}$. Observe, that if $\hat{u}_{i}$ is $\overline{\boldsymbol{\theta}}$-uniformly $\tau$-proper for a Hausdorff locally convex topology $\tau$ on $\phi$, then (in view of $\tau \subseteq \xi$ ) $\hat{u}_{i}$ is automatically $\overline{\boldsymbol{\theta}}$-uniformly $\boldsymbol{\xi}$-proper.

We have the following.

Theorem 6.1: Assume that the cone of positive payoff portfolios $\phi_{R}^{+}$has a Yudin basis, and that each portfolio utility function $\hat{u}_{i}$ is also $\overline{\boldsymbol{\theta}}$-uniformly $\xi$-proper on $\phi_{R}^{+}$. Then there exists a portfolio quasiequilibrium.

If $\hat{u}_{i}$ is the indirect utility function $u_{i}(R(\cdot))$ obtained from the consumption utility function $u_{i}: \mathrm{X}^{+} \rightarrow \mathbb{R}$ which is $\overline{\mathrm{X}}$-uniformly $\tau$-proper for a Hausdorff locally convex topology $\tau$ on $\mathrm{X}$ (where $\overline{\mathrm{x}}=R(\overline{\boldsymbol{\theta}})$ ), then $\hat{u}_{i}$ is $\overline{\boldsymbol{\theta}}$-uniformly $\xi$-proper on $\phi_{R}^{+}$. Properness of consumption utility functions is a standard assumption in infinite dimensional general equilibrium theory; see, for instance Aliprantis et al. (1990) or Mas-Colell (1986).

Corollary 6.1: Assume that the positive cone $M^{+}$of the asset span has a Yudin basis, and that each consumption utility function $u_{i}$ is continuous and $\overline{\mathrm{x}}$-uniformly proper for a Hausdorff locally convex topology on X. Then there exists a portfolio quasiequilibrium. 


\section{Equivalent market structures}

Any sequence $\left\{\mathrm{x}_{n}\right\}$ of linearly independent payoffs defines a market structure. Associated with the market structure $\mathrm{x}=\left\{\mathrm{x}_{n}\right\}$ is an asset span $M_{\mathrm{x}}$, the payoff operator $R_{\mathrm{x}}: \phi \rightarrow M_{\mathrm{x}}$ defined by $R_{\mathrm{x}}(\boldsymbol{\theta})=\sum_{n=1}^{\infty} \boldsymbol{\theta}_{n} \mathrm{x}_{n}$, a portfolio dominance order $\geq_{R_{\mathrm{x}}}$, and a cone of positive payoff portfolios $\phi_{R_{\mathrm{x}}}^{+}$. For simplicity, we shall denote the portfolio dominance order $\geq_{R_{\mathrm{x}}}$ by $\geq_{\mathrm{x}}$ and the cone of positive payoff portfolios $\phi_{R_{\mathrm{x}}}^{+}$by $\phi_{\mathrm{x}}^{+}$.

Two market structures that give rise to the same asset span provide investors with the same opportunities of insuring against the consumption risk and will be referred to as equivalent market structures.

Definition 7.1: Two market structures $\mathrm{x}=\left\{\mathrm{x}_{n}\right\}$ and $z=\left\{z_{n}\right\}$ are equivalent if they have the same asset span, i.e., if $M_{\mathrm{x}}=M_{z}$.

If market structures $\mathrm{x}=\left\{\mathrm{x}_{n}\right\}$ and $z=\left\{z_{n}\right\}$, are equivalent, then a linear operator $\Phi: \phi \rightarrow \phi$ is naturally defined via the formula

$$
\Phi(\boldsymbol{\theta})=R_{z}^{-1}\left(R_{\mathrm{x}}(\boldsymbol{\theta})\right) .
$$

The operator $\Phi:\left(\phi, \phi_{\mathrm{x}}^{+}\right)\left(\phi, \phi_{z}^{+}\right)$is an order isomorphism between these two partially ordered portfolio spaces. This implies that the portfolio dominance $\geq_{\mathrm{x}}$ is a lattice order if and only if $z_{z}$ is likewise a lattice order-and this is equivalent to assuming that $M^{+}$is a lattice cone of $M=M_{\mathrm{x}}=M_{z}$ (or that $M$ is a lattice-subspace of X).

Thus the property of portfolio dominance being a lattice order is independent of the market structure as long as market structures are equivalent, and $M$ is a lattice-subspace of X. Furthermore, the cone of positive payoff portfolios $\phi_{\mathrm{x}}^{+}$has a Yudin basis if and only if the cone $\phi_{z}^{+}$has a Yudin basis, and this is, of course, equivalent to requiring that $M^{+}$has a Yudin basis.

The operator $\Phi:\left(\phi, \phi_{\mathrm{x}}^{+}, \xi\right) \rightarrow\left(\phi, \phi_{z}^{+}, \xi\right)$ is also a topological order isomorphism. The adjoint operator $\Phi^{\prime}$ is therefore a well-defined positive operator. It maps the space of security prices $\mathbb{R}^{\infty}$ into itself and is given via the duality identity

$$
\Phi^{\prime}(\mathrm{q}) \cdot \boldsymbol{\theta}=\mathrm{q} \cdot \Phi(\boldsymbol{\theta})
$$

for $\boldsymbol{\theta} \in \phi$ and $\mathrm{q} \in \mathbb{R}^{\infty}$. In case $M$ has a Yudin basis, then the adjoint operator $\Phi^{\prime}:\left(\mathbb{R}^{\infty},\left(\phi_{z}^{+}\right)^{\prime}\right)\left(\mathbb{R}^{\infty},\left(\phi_{\mathrm{x}}^{+}\right)^{\prime}\right)$ is a (surjective) lattice isomorphism.

Suppose that each investor's portfolio utility functions $\hat{u}_{i}^{\mathrm{x}}: \phi_{\mathrm{x}}^{+} \rightarrow \mathbb{R}$ and $\hat{u}_{i}^{z}: \phi_{z}^{+}$ $\rightarrow \mathbb{R}$ are indirect utilities of a consumption utility function $u_{i}$ given by $\hat{u}_{i}^{\mathrm{x}}(\boldsymbol{\theta})=$ $u_{i}\left(R_{\mathrm{x}}(\boldsymbol{\theta})\right)$ and $\hat{u}_{i}^{z}(\boldsymbol{\theta})=u_{i}\left(R_{z}(\boldsymbol{\theta})\right)$. Furthermore, let the initial portfolios $\overline{\boldsymbol{\theta}}_{\mathrm{x}}^{i}$ and $\overline{\boldsymbol{\theta}}_{z}^{i}$ be such that $R_{\mathrm{x}}\left(\overline{\boldsymbol{\theta}}_{\mathrm{x}}^{i}\right)=R_{z}\left(\overline{\boldsymbol{\theta}}_{z}^{i}\right)$, i.e., they have the same payoff. The duality properties of the operator $\Phi$ allow us to state an interesting invariance result. 
Theorem 7.1: For two equivalent market structures $\mathrm{x}=\left\{\mathrm{x}_{n}\right\}$ and $z=\left\{z_{n}\right\}$ and portfolios $\boldsymbol{\theta}_{1}, \ldots, \boldsymbol{\theta}_{m} \in \phi$ we have the following invariance results:

1. $\left(\boldsymbol{\theta}_{1}, \ldots, \boldsymbol{\theta}_{m}\right)$ is an optimal portfolio allocation with respect to utility functions $\hat{u}_{i}^{\mathrm{x}}$ if and only if $\left(\Phi\left(\boldsymbol{\theta}_{1}\right), \ldots, \Phi\left(\boldsymbol{\theta}_{m}\right)\right)$ is an optimal portfolio allocation with respect to utility functions $\hat{u}_{i}^{z}$.

2. $\left(\boldsymbol{\theta}_{1}, \ldots, \boldsymbol{\theta}_{m}\right)$ is a portfolio equilibrium with respect to a price $\mathrm{q} \in \mathbb{R}^{\infty}$ relative to the market structure $\mathrm{x}$ if and only if $\left(\Phi\left(\boldsymbol{\theta}_{1}\right), \ldots, \Phi\left(\boldsymbol{\theta}_{m}\right)\right)$ is a portfolio equilibrium relative to the market structure $z$ with respect to the price $\left(\Phi^{\prime}\right)^{-1}(\mathrm{q})$.

The proof is straightforward and therefore omitted. Note that (1) implies that the utility set is independent of the market structure.

Suppose that the market structure $\left\{\mathrm{x}_{n}\right\}$ is such that the asset span $M_{\mathrm{x}}$ is a lattice-subspace of $\mathrm{X}$, and that the positive cone $M_{\mathrm{x}}^{+}$has a Yudin basis. By Theorem 6.1 these properties guarantee the existence of optimal portfolio allocations and the existence of portfolio quasi-equilibria.

Let $\left\{f_{n}\right\}$ be the Yudin basis of $M_{\mathrm{x}}^{+}$. Each payoff $f_{n}$ is the payoff of some portfolio $\eta^{n} \in \phi$, i.e., $f_{n}=R\left(\eta^{n}\right)$. Note that portfolio $\eta^{n}$ may involve short (i.e., negative) positions in some securities. The portfolios $\eta^{n}$ can be thought of as mutual funds. The market structure $\left\{f_{n}\right\}$ is equivalent to the market structure $\left\{\mathrm{x}_{n}\right\}$. Therefore trading the mutual funds provides the same spanning opportunities as trading the original securities.

By the definition of Yudin basis, the portfolio dominance order $\geq_{f}$ associated with the market structure $\left\{f_{n}\right\}$ coincides with the standard pointwise order $\geq$ of the portfolio space $\phi$. Consequently, the cone $\phi_{f}^{+}$of portfolios of mutual funds with positive payoffs equals the standard positive cone $\phi^{+}$. A portfolio of mutual funds has a positive payoff if and only if the share-holding of each fund is positive. The restriction of positive wealth is therefore equivalent to the restriction of no short sales of mutual funds. For an investor who plans to have positive consumption, the restriction of no short sales of mutual funds is nonbinding. The condition of the existence of a Yudin basis of the positive cone $\mathrm{M}^{+}$which played a crucial role in our analysis in Sections 5 and 6 can be given the following simple interpretation: There exist mutual funds such that the restriction of no short sales of mutual funds is nonbinding.

\section{The portfolio-consumption duality}

Our analysis of security markets thus far has been focused on portfolio allocations and security prices. Portfolio equilibria and optimal portfolio allocations have their counterparts in the payoff space. There is a simple duality between portfolios and consumption plans, and between security prices and consumption prices. This duality is the subject of this section. 
For an investor who trades in security markets, only consumption plans in the asset span $M$ are feasible. Consumption plans in $M$ will be referred to as constrained consumption plans. The asset span $M$ is a vector space with positive cone $M^{+}=M \cap \mathrm{X}^{+}$. We equip $M$ with its inductive limit topology. Every linear functional on $M$ is continuous in the inductive limit topology, i.e., the topological dual of $M$ coincides with the algebraic dual. Let $M^{\prime}$ be the space of all linear functionals on $M$. Each functional $p$ in $M^{\prime}$ is a constrained consumption price system. The value of constrained consumption $\mathrm{y} \in M$ under the price $p$ is $p \cdot \mathrm{y}$.

The dual system $\left\langle M, M^{\prime}\right\rangle$ is the constrained consumption-price duality. Between the portfolio-price dual system and the consumption-price dual system we have the payoff operator that maps portfolios into constrained consumptions, and the adjoint payoff operator that maps consumption prices into security prices. The payoff operator is continuous in the inductive limit topologies of $\phi$ and $M$, and therefore the adjoint payoff operator $R^{\prime}$ is well-defined.

A constrained consumption allocation is any vector $\left(\mathrm{y}_{1}, \ldots, \mathrm{y}_{m}\right)$ with $\mathrm{y}_{i} \in M^{+}$ for each $i$, and $\sum_{i=1}^{m} \mathrm{y}_{i}=\overline{\mathrm{x}}=R(\overline{\boldsymbol{\theta}})$.

Definition 8.1: A constrained consumption allocation $\left(\mathrm{y}_{1}, \ldots, \mathrm{y}_{m}\right)$ is said to be a constrained consumption equilibrium, if there exists a non-zero price system $p \in M^{\prime}$ such that each $\mathrm{y}_{i}$ maximizes $u_{i}(\mathrm{x})$ subject to $\mathrm{x} \in M^{+}$and $p \cdot \mathrm{x} \leq p \cdot R\left(\overline{\boldsymbol{\theta}}^{i}\right)$.

It follows easily from duality relations that portfolio equilibria are in duality with the constrained consumption equilibria.

Proposition 8.1: We have the following:

1. If $\left(\mathrm{y}_{1}, \ldots, \mathrm{y}_{m} ; p\right)$ is a constrained consumption equilibrium, then $\left(R^{-1}\left(\mathrm{y}_{1}\right) \ldots, R^{-1}\left(\mathrm{y}_{m}\right) ; R^{\prime}(p)\right)$ is a portfolio equilibrium.

2. If $\left(\boldsymbol{\theta}_{1}, \ldots, \boldsymbol{\theta}_{m} ; R^{\prime}(p)\right)$ is a portfolio equilibrium, then $\left(R\left(\boldsymbol{\theta}_{1}\right), \ldots, R\left(\boldsymbol{\theta}_{m}\right) ; p\right)$ is a consumption equilibrium.

A constrained optimal consumption allocation is defined as follows.

Definition 8.2: A constrained consumption allocation $\left(\mathrm{y}_{1}, \ldots, \mathrm{y}_{m}\right)$ is said to be constrained optimal, if there is no other constrained consumption allocation $\left(z_{1}, \ldots, z_{m}\right)$ satisfying $u_{i}\left(z_{i}\right) \geq u_{i}\left(\mathrm{y}_{i}\right)$ for every $i$ and $u_{i}\left(z_{i}\right)>u_{i}\left(\mathrm{y}_{i}\right)$ for at least one $i$.

Of course, a constrained optimal allocation need not be Pareto optimal among all consumption allocations in X. It is easily seen that optimal portfolio allocations are in duality with the constrained consumption allocations.

Proposition 8.2: A constrained consumption allocation $\left(\mathrm{y}_{1}, \ldots, \mathrm{y}_{m}\right)$ is constrained optimal if and only if the portfolio allocation $\left(\boldsymbol{\theta}^{l}, \ldots, \boldsymbol{\theta}^{m}\right)$, where $R\left(\boldsymbol{\theta}^{i}\right)=\mathrm{y}_{i}$ for every $i$, is an optimal portfolio allocation. 


\section{Acknowledgements}

The research of C.D. Aliprantis and I.A. Polyrakis was partially supported by the 1995 PENED Program of the Ministry of Industry, Energy and Technology of Greece and by the NATO Collaborative Research Grant \#941059. Roko Aliprantis also expresses his deep appreciation for the hospitality provided by the Department of Economics and the Center for Analytic Economics at Cornell University and the Division of Humanities and Social Sciences of the California Institute of Technology where part of the paper was written during his sabbatical leave (January to June, 1996). J. Werner acknowledges the financial support of the Deutsche Forschungsgemainschaft, SFB 303, University of Bonn during his sabbatical leave August 1995 to July 1996.

\section{References}

Abramovich, Y.A., Aliprantis, C.D., Polyrakis, I.A., 1994. Lattice-subspaces and positive projections. Proc. R. Irish Acad. 94, 237-253.

Aliprantis, C.D., 1996. Problems in Equilibrium Theory. Springer-Verlag, New York.

Aliprantis, C.D., 1996. Separable utility functions. Center for Analytic Economics, Cornell University, Working Paper \#96-02, forthcoming in J. Mathematical Economics 28.

Aliprantis, C.D., Border, K.C., 1994. Infinite Dimensional Analysis, Studies in Economic Theory, \#4. Springer-Verlag, Heidelberg.

Aliprantis, C.D., Burkinshaw, O., 1978. Locally Solid Riesz Spaces. Academic Press, New York.

Aliprantis, C.D., Burkinshaw, O., 1985. Positive Operators. Academic Press, New York.

Aliprantis, C.D., Brown, J., Burkinshaw, O., 1990. Existence and Optimality of Competitive Equilibria. Springer-Verlag, Heidelberg.

Aliprantis, C.D., Brown, D.J., Polyrakis, I.A., Werner, J., 1996. Yudin cones and inductive limit topologies. Caltech Social Science Working Paper \#964. Forthcoming in Atti Sem. Mat. Fis. University, Modena.

Aliprantis, C.D., Brown, D.J., Werner, J., May 1996. Hedging with derivatives in incomplete markets. Cowles Foundation Discussion Paper \#1126, Yale University.

Brown, D.J., Werner, J., 1995. Arbitrage and existence of equilibrium in infinite asset markets. Rev. Economic Studies 62, 101-114.

Cheng, H., 1991. Asset market equilibrium in infinite dimensional complete markets. J. Mathematical Economics 20, 137-152.

Chichilnisky, G., Heal, G.M., 1993. Competitive equilibrium in Sobolev spaces without bounds on short sales. J. Economic Theory 59, 364-384.

Connor, G., 1984. A unified beta pricing theory. J. Economic Theory 34, 13-31.

Dana, R.A., Le Van, C., 1996. Asset Equilibria in $L^{p}$-Spaces with Complete Markets: A Duality Approach. J. Mathematical Economics 25, 263-280.

Dana, R.A., Le Van, C., Magnien, F., 1994. General equilibrium in asset markets with and without short-selling. Working J. Mathematical Analysis and Applications 206, 567-588.

Hammond, P., 1983. Overlapping expectations and Hart's condition for equilibrium in securities model. J. Economic Theory 31, 170-175.

Harrison, J.M., Kreps, D.M., 1979. Martingales and arbitrage in multiperiod securities markets. J. Economic Theory 20, 381-408. 
Hart, O.D., 1974. On the existence of equilibrium in a securities model. J. Economic Theory 9, 293-311.

Ishimoto, O., 1996. Equilibrium in Infinite Asset Markets with Short Selling. University of Windsor.

Luxemburg, W.A.J., Zaanen, A.C., 1971. Riesz Spaces. North-Holland, Amsterdam.

Mas-Colell, A., 1986. The price equilibrium existence problem in topological vector lattices. Econometrica 54, 1039-1053.

Milne, F., 1976. Default risk in a general equilibrium asset economy with incomplete markets. Int. Economic Rev. 17, 613-625.

Milne, F., 1987. The induced preference approach to arbitrage and diversification arguments in finance. Eur. Economic Rev. 31, 235-245.

Miyajima, S., 1983. Structure of Banach quasi-sublattices. Hokkaido Mathematical J. 12, 83-91.

Nielsen, L.T., 1989. Asset market equilibrium with short-selling. Rev. Economic Studies 56, 467-474.

Page, F., 1987. On equilibrium in Hart's securities exchange model. J. Economic Theory 41, 392-404.

Polyrakis, I.A., 1994. Lattice-subspaces of $C[0,1]$ and positive bases. J. Mathematical Analysis Applications 184, 1-18.

Polyrakis, I.A., 1996. Finite-dimensional lattice-subspaces of $C(\Omega)$ and curves of $\mathbb{R}^{\mathrm{n}}$. Trans. Am. Mathematical Soc. 348, 2793-2810.

Ross, S.A., 1976. The arbitrage theory of capital asset pricing. J. Economic Theory 13, 341-360.

Werner, J., 1987. Arbitrage and the existence of competitive equilibrium. Econometrica 55, 1403-1418.

Werner, J., 1997. Diversification and equilibrium in securities markets. Technical Report, SITE, J. Economic Theory 75, 89-103. 